\title{
When to CHEUS Contrast-Enhanced Endoscopic Ultrasound to Assess Gallbladder Wall Thickening
}

\author{
Gareth Morris-Stiff $\cdot$ Shahid Farid
}

Received: 29 May 2014/ Accepted: 29 May 2014/Published online: 7 June 2014

(C) Springer Science+Business Media New York 2014

Gallbladder wall thickening identified on trans-abdominal ultrasound is a relatively common finding that can be due to common biliary pathologies such as cholecystitis, gallbladder carcinoma, and adenomyomatosis, while systemic disease and extracholecystic inflammation may also distort gallbladder morphology [1]. In many cases, ultrasound imaging combined with the clinical history will suffice; however, for some patients, further imaging studies are required in order to accurately distinguish benign and malignant pathologies. At the extremes, the patient with a suspected gallbladder cancer requires urgent referral to a specialist hepatobiliary surgeon whereas the elderly, frail patient with adenomyomatosis may simply be reassured.

Cross-sectional imaging with computed tomography (CT) or magnetic resonance imaging (MRI) can provide valuable additional information concerning the etiology of gallbladder wall thickening. However, there are surprisingly few studies that have addressed these modalities in the context of gallbladder wall thickening [2-5], even though current cross-sectional imaging modalities can accurately differentiate benign from malignant pathologies in the majority of cases. When these techniques reveal equivocal results, endoscopic ultrasound (EUS) may be considered. Despite its widespread use in the evaluation of pancreatobiliary disease, it has not yet gained widespread

\section{G. Morris-Stiff ( $\square)$}

Division of Hepato-Pancreato-Biliary Surgery, Department of General Surgery, A100 Cleveland Clinic Foundation, Cleveland, OH 44195, USA

e-mail: garethmorrisstiff@hotmail.com

\section{S. Farid}

Department of Hepato-Pancreato-Biliary and Transplantation Surgery, St James's University Hospital, Beckett Street, Leeds LS9 7TF, UK use in evaluating the gallbladder $[6,7]$. In the setting of a thickened gallbladder wall, Kim and colleagues reported it to be of value with a wall thickness $>10 \mathrm{~mm}$ and reduced internal echogenicity being predictive of malignancy [8].

In this issue of Digestive Diseases and Sciences, Imazu et al. [9] evaluated contrast-enhanced harmonic endoscopic ultrasonography (CH-EUS) using a second-generation contrast agent consisting of perfluorobutane microspheres (Sonazoid) in the diagnosis of patients exhibiting gallbladder wall thickening. The group examined 36 patients with equivocal conventional ultrasound and cross-sectional imaging in whom surgery was performed to provide histopathologic confirmation. The performance characteristics (sensitivity, specificity, accuracy, positive predictive value, negative predictive value, and area under the receiver operator curve) of CH-EUS were 89.6, 98, 94.4, 97.7, 92.2, and 0.94, respectively, superior to conventional EUS in each case. The authors assessed inter-observer variation by comparing assessments made by an experienced endosonographer, a trainee EUS operator, and an experienced gastroenterologist, demonstrating an improvement in $\mathbf{K}$ score from 0.51 (moderate) to 0.77 (substantial) with the addition of Sonazoid. They also identified that an inhomogeneous enhancement pattern on $\mathrm{CH}$-EUS, and a wall thickness $>12 \mathrm{~mm}$ without contrast were strongly predictive of a malignant etiology.

CH-EUS is based on harmonic imaging, which exploits the nonlinear propagation of acoustic signals through biological tissues, with secondary signals generated by the tissue creating high-resolution images. The use of contrast provides the key advantage of enabling visualization of the microvasculature in real time [9].

Although this is the first series evaluating $\mathrm{CH}$-EUS in assessing gallbladder wall thickness, the technique has been used with success in the evaluation of gallbladder [10, 11] polyps and pancreatic lesions [12]. 
Despite the many advantages of $\mathrm{CH}$-EUS reported by Imazu et al., there are several issues requiring further clarification. First, the details of cross-sectional imaging were not provided. Second, no comparison was made between the performance characteristics of CT/MRI and that of $\mathrm{CH}$-EUS, therefore raising the possibility of a selection bias. In order for the technique to gain widespread use, its characteristics need to be better defined with larger studies incorporating formal comparison of crosssectional and endoscopic imaging. If CT and MRI were applied sequentially using contemporary protocols, it is likely that only a small proportion of patients would require $\mathrm{CH}$-EUS. Thus, the value of CH-EUS should be assessed in a population, in which these more conventional imaging techniques failed to establish a diagnosis.

Although the degree of inter-observer agreement was high in the discussed article [9], it is clear that CH-EUS, as with conventional EUS, requires a high degree of operator expertise in order to maximize its clinical value. Limiting the technique to specialist referral centers would appear prudent pending its widespread adoption.

A final issue is the availability of the contrast agent. At present, it does not have approval by the United States Food and Drug Administration for evaluation of the biliary tree and pancreas. Given its enhancements to the performance characteristics of EUS, full adoption of the technique in the US awaits is testing and approval.

In conclusion, $\mathrm{CH}$-EUS shows considerable promise in the diagnosis of the etiology of gallbladder wall thickening in subjects with equivocal results of trans-abdominal ultrasonography and cross-sectional imaging. Widespread study of the technique in large populations combined with full regulatory approval of associated specialized contrast agents will be needed in order to establish the technique as a useful tool in the armamentarium of advanced hepatobiliary endoscopists.

\section{References}

1. Van Breda Vriesman AC, Engelbrecht MR, Smithuis RHM, Puylaert JBCM. Diffuse gallbladder wall thickening: differential diagnosis. AJR. 2007;188:495-501.

2. Ching BH, Yeh BM, Westphalen AC, Joe BN, Qayyum A, Coakley FV. CT differentiation of adenomyomatosis and gallbladder cancer. AJR. 2007;189:62-66.

3. Kim SJ, Lee JM, Lee JY, et al. Analysis of enhancement pattern of flat gallbladder wall thickening on MDCT to differentiate gallbladder cancer from cholecystitis. AJR. 2008;191:765-771.

4. Jung SE, Lee JM, Lee K, et al. Gallbladder wall thickening: MR imaging and pathologic correlation with emphasis on layered pattern. Eur Radiol. 2005;15:694-701.

5. Lee NK, Kim S, Kim TU, Kim DU, Seo HI, Jeon TY. Diffusionweighted MRI for differentiation of benign from malignant lesions in the gallbladder. Clin Radiol. 2014;69:e78-e85.

6. Muguruma N, Okamura S, Ichikawa S, et al. Endoscopic sonography in the diagnosis of gallbladder wall lesions in patients with gallstones. J Clin Ultrasound. 2001;29:395-400.

7. Kim MY, Baik SK, Choi YJ, et al. Endoscopic sonographic evaluation of the thickened gallbladder wall in patients with acute hepatitis. J Clin Ultrasound. 2003;31:245-249.

8. Kim HJ, Park JH, Park DI, et al. Clinical usefulness of endoscopic ultrasonography in the differential diagnosis of gallbladder wall thickening. Dig Dis Sci. 2012;57:508-515.

9. Imazu H, Mori N, Kanazawa K et al. Contrast-enhanced harmonic endoscopic ultrasonography in the differential diagnosis of gallbladder wall thickening. Dig Dis Sci. (Epub ahead of print). doi:10.1007/s10620-014-3115-5.

10. Choi JH, Seo DW, Choi JH, et al. Utility of contrast-enhanced harmonic EUS in the diagnosis of malignant gallbladder polyps (with videos). Gastrointest Endosc. 2013;78:484-493.

11. Park CH, Chung MJ, Oh TG, et al. Differential diagnosis between gallbladder adenomas and cholesterol polyps on contrastenhanced harmonic endoscopic ultrasonography. Surg Endosc. 2013;27:1414-1421.

12. Park JS, Kim HK, Bang BW, Kim SG, Jeong S, Lee DH. Effectiveness of contrast-enhanced harmonic endoscopic ultrasound for the evaluation of solid pancreatic masses. World $J$ Gastroenterol. 2014;20:518-524. 